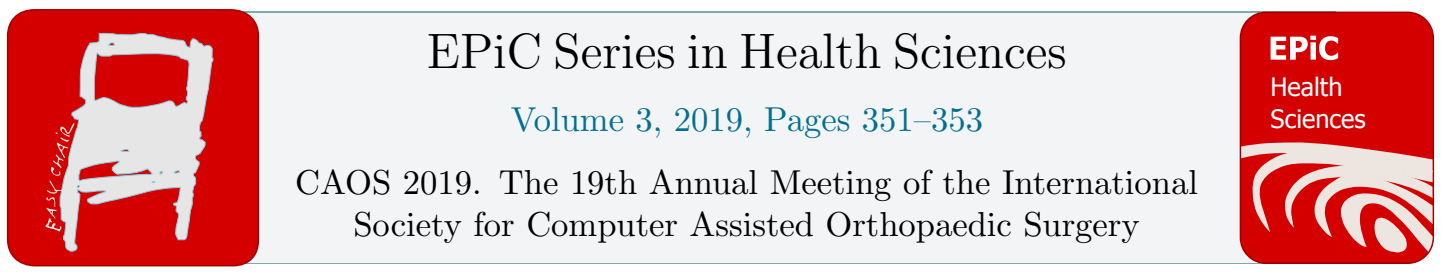

\title{
Robotic Assisted Total Knee Arthroplasty in Presence of Extra- Articular Deformity
}

\author{
Nipun Sodhi ${ }^{1}$; Anton Khlopas²; Joseph O. Ehioroboํㅜ Caitlin Condrey ${ }^{3}$; Robert C. \\ Marchand $^{3}$; Matthew S. Hepinstall ${ }^{1}$; Michael A. Mont ${ }^{1}$ \\ ${ }^{1}$ Lenox Hill Hospital, Northwell Health, New York, New York \\ ${ }^{2}$ Cleveland Clinic, Cleveland, Ohio \\ ${ }^{3}$ Ortho Rhode Island, Fort Lauderdale, Florida
}

\section{Introduction:}

Tibial or femoral extra-articular deformities complicate the goal achieving optimal mechanical axis alignment for successful total knee arthroplasty (TKA) outcomes. In the presence of these extra-articular deformities, standard operative techniques and instruments may not be reliable. Robotic-arm assisted technology was developed to help achieve a well-aligned and balanced knee in a variety of clinical scenarios ${ }^{1-4}$. Although prior case series have reported on the use of robotic-arm assisted devices for cases with severe angular deformity, there is a lack of data concerning the use of the robotic device for patients with other potentially complex surgical factors. Therefore, the purpose of this series was to present cases in which the robotic-arm assisted TKA application was used in the setting of extra-articular deformities to educate the surgeon community on this potentially useful method to address these complex cases.

\section{Methods:}

Three cases with of patients who underwent robotic-arm assisted TKA in the setting of pre-operative extra-articular deformities were identified. These included one with femoral and tibial fracture malunion, another with a proximal tibial fracture nonunion, and another with a healed tibial plateau fracture. Patient clinical histories, intra-operative surgical techniques, and post-operative outcomes were obtained. Specific focus was placed on the surgical management of the patient's pre-existing deformity. 


\section{Results:}

These three case reports are discussed in detail, with emphasis on pre-operative planning and intra-operative techniques. The robotic software was able to appropriately consider the extra-articular deformity in the pre-operative and real-time updated intra-operative plans. Doing so, the surgeon was able to achieve balanced and aligned TKA in each case. All three patients underwent robotic assisted total knee arthroplasty with no intra-operative or post-operative complications. For all patients, their antero-posterior and lateral radiographs demonstrated well fixed and aligned femoral and tibial components with no signs of loosening or osteolysis. On physical exam, all patients had excellent range of motion with mean flexion of 122 degrees (range: 120 to 125 degrees of flexion) at final follow-up.

\section{Discussion:}

The decision on how to best approach TKA in patients with extra-articular deformity should be based on an extensive patient history, physical examination, and thorough evaluation of the magnitude and proximity of the deformity to the knee joint. Utilizing pre-operative CT-scans with a 3D plan for robotic-arm assisted surgery allowed for appropriate assessment of the deformity pre-operatively and execution of a plan for a balanced and aligned total knee arthroplasty ${ }^{2,5-11}$. We have demonstrated excellent results utilizing robotic-arm assisted TKA in these complex cases.

\section{References}

1. Marchand RC, Sodhi N, Khlopas A, Sultan AA, Higuera CA, Stearns KL, et al. Coronal Correction for Severe Deformity Using Robotic-Assisted Total Knee Arthroplasty. J Knee Surg Germany, 2018;31(1):2-5.

2. Hampp EL, Chughtai M, Scholl LY, Sodhi N, Bhowmik-Stoker M, Jacofsky DJ, et al. Robotic-Arm Assisted Total Knee Arthroplasty Demonstrated Greater Accuracy and Precision to Plan Compared with Manual Techniques. J Knee Surg Germany, 2018;

3. Hampp EL, Chughtai M, Scholl LY, Bhowmik-Stoker M, Jacofsky DJ, Mont MA. Robotic-Arm Assisted Total Knee Arthroplasty Demonstrated Improved Accuracy and Precision Compared to Manual Technique. J Knee Surg 2017;Submitted.

4. Marchand R, Khlopas A, Sodhi N, Condrey C, Piuzzi N, Patel R, et al. Difficult Cases in Robotic Arm-Assisted Total Knee Arthroplasty: A Case Series. J Knee Surg [Internet] 2017 [cited 27 Nov 2017];

5. Petursson G, Fenstad AM, Gothesen O, Dyrhovden GS, Hallan G, Rohrl SM, et al. Computer-Assisted Compared with Conventional Total Knee Replacement: A Multicenter Parallel-Group Randomized Controlled Trial. J 
Bone Joint Surg Am United States, 2018;100(15):1265-1274.

6. Bae DK, Song SJ. Computer Assisted Navigation in Knee Arthroplasty. Clin Orthop Surg [Internet] The Korean Orthopaedic Association, 2011;3(4):259267.

7. HARITINIAN EG, PIMPALNERKAR AL. Computer Assisted Total Knee Arthroplasty: Does it Make a Difference? Mcedica [Internet] Media Med Publicis, 2013;8(2):176-181.

8. Lee CM, Dhillon MK, Sulaiman MA. A computer-assisted, tibia-first technique for improved femoral component rotation in total knee arthroplasty. Arthroplast today United States, 2018;4(1):78-84.

9. Mota e Albuquerque RF da. NAVIGATION IN TOTAL KNEE ARTHROPLASTY. Rev Bras Ortop [Internet] Elsevier, 2011;46(1):18-22.

10. Khlopas A, Sodhi N, Sultan AA, Chughtai M, Molloy RM, Mont MA. Robotic Arm-Assisted Total Knee Arthroplasty. J Arthroplasty 2018;20022006.

11. Khlopas A, Chughtai M, Hampp EL, Scholl LY, Prieto M, Chang T-C, et al. Robotic-Arm Assisted Total Knee Arthroplasty Demonstrated Soft Tissue Protection. Surg Technol Int 2017;30:441-446. 